\title{
High Pallidal T1 Signal is Rarely Observed in Obstructive Jaundice, but is Frequently Observed in Liver Cirrhosis
}

\author{
Sung-Jo BAng ${ }^{1}$, Seong Hoon $\mathrm{CHOI}^{2}$, Neung Hwa PARK ${ }^{1}$, Jung Woo Shin ${ }^{1}$, Nari ChOY ${ }^{3}$, \\ Suk Hwan $\mathrm{K}_{\mathrm{Im}}{ }^{3}$ and Yangho $\mathrm{K}_{\mathrm{Im}}{ }^{3}$
}

${ }^{1}$ Department of Internal Medicine, ${ }^{2}$ Department of Radiology and ${ }^{3}$ Department of Occupational and Environmental Medicine, Ulsan University Hospital, University of Ulsan College of Medicine, South Korea

\begin{abstract}
High Pallidal T1 Signal is Rarely Observed in Obstructive Jaundice, but is Frequently Observed in Liver Cirrhosis: Sung-Jo BANG, et al. Department of Internal Medicine, Ulsan University Hospital, University of Ulsan College of Medicine, South Korea-Although high signal intensities in the globus pallidus are frequently observed in T1-weighted magnetic resonance images (MRI) of patients with liver cirrhosis, it is unclear whether these increases are due to portal-systemic shunt or obstruction of biliary excretion. We therefore studied pallidal signals in 18 cancer patients with bile duct obstruction and marked jaundice (>10 mg/d $/$ ). Patients who had fever, leukocytosis or liver cirrhosis were excluded to ensure that jaundice was due to bile duct obstruction. All patients showed a dilated intrahepatic duct on computed tomography (CT) scan. A high pallidal signal was observed in one of 18 biliary obstructive patients whereas high signals were highly prevalent in liver cirrhosis. A portal-systemic shunt rather than an obstruction of biliary excretion may be responsible for the increased blood manganese concentrations and pallidal T1 signals in chronic liver diseases.
\end{abstract}

(J Occup Health 2007; 49: 268-272)

Key words: Manganese, Signal intensity, Magnetic resonance, Liver cirrhosis, Biliary obstruction, Pallidal index

Symmetrical increases in signal intensities, mainly confined to the globus pallidus (GP) and midbrain, can be observed on T1-weighted brain magnetic resonance images (MRI) due to the paramagnetic property of

Received Feb 22, 2007; Accepted Apr 12, 2007

Correspondence to: Y. Kim, Department of Occupational and Environmental Medicine, Ulsan University Hospital, \#290-3 Cheonha-Dong, Dong-Gu, Ulsan 682-060, South Korea (e-mail: yanghokm@nuri.net) manganese (Mn), whereas T2-weighted images are not altered. Characteristic high signal intensities, mainly confined to the GP, have also been observed in T1weighted MRI during experimental Mn poisoning of nonhuman primates ${ }^{1)}$ and in a patient with $\mathrm{Mn}$ neurointoxication ${ }^{2}$. A similar MRI pattern has been observed in patients receiving total parenteral nutrition, because of excessive $\mathrm{Mn}$ intake ${ }^{3,4)}$. Chronic liver failure is also associated with increased T1-weighted signal intensities in the $\mathrm{GP}^{5,6}$. The correlation between signal intensities in the GP and circulating Mn concentrations of cirrhotic patients suggests that the high signals are due to paramagnetic $\mathrm{Mn}^{7-9}$. Although high signal intensities in the GP have also been reported to be highly prevalent in patients with liver cirrhosis $(52-100 \%)^{10-12)}$, it is unclear whether the increases in pallidal signals are due to portal-systemic shunt or obstruction of biliary excretion. Studying patients with biliary obstruction without liver cirrhosis may therefore clarify the role of biliary obstruction in increased pallidal $\mathrm{T} 1$ signals. We therefore assessed pallidal T1 signals in 18 cancer patients who had bile duct obstruction and marked jaundice without liver cirrhosis, and compared these findings with pallidal signals in patients with liver cirrhosis and healthy controls.

\section{Subjects and Methods}

Subjects

We selected 18 cancer patients, with biliary duct, pancreatic head, or gall bladder cancer, who had bile duct obstruction and marked jaundice $(>10 \mathrm{mg} / \mathrm{d} l)$ without liver cirrhosis. Patients with cholangitis (fever and leukocytosis), anemia or liver cirrhosis were excluded to ensure that the jaundice was due to bile duct obstruction. Patients with calcification in the GP, which affects pallidal signals, were also excluded. All patients showed a dilated intrahepatic duct on computed tomography (CT) scan. We also selected 29 patients with clinically confirmed 
Table 1. Demographic, clinical and laboratory features of the studied subjects

\begin{tabular}{|c|c|c|c|}
\hline & Healthy controls $(n=16)$ & Liver cirrhotics $(\mathrm{n}=29)$ & $\begin{array}{l}\text { Biliary obstructive } \\
\text { patients }(n=18)\end{array}$ \\
\hline Mean age (years) & $53.8 \pm 10.5^{*}$ & $47.5 \pm 5.9^{*}$ & $66.8 \pm 9.3$ \\
\hline Proportion of males (\%) & 93.8 & 100 & $66.7 \&$ \\
\hline $\begin{array}{l}\text { Proportion of drinkers }(\%) \\
\text { (premorbid) }\end{array}$ & 56.3 & 72.4 & 38.9 \\
\hline $\begin{array}{l}\text { Proportion of persons with } \\
\text { high signal intensity on MRI }(\%)\end{array}$ & 0.0 & 86.2 & $5.6^{\&}$ \\
\hline Pallidal indices & $\begin{array}{c}101.84 \pm 2.98^{\dagger} \\
(95.56-106.59)\end{array}$ & $\begin{array}{c}125.63 \pm 12.49 \\
(100.45-153.05)\end{array}$ & $\begin{array}{c}100.77 \pm 4.30^{\dagger} \\
(94.51-109.82)\end{array}$ \\
\hline $\operatorname{MnB}(\mu \mathrm{g} / \mathrm{d} l)$ & $1.20 \pm 0.30(0.58-1.59)^{\dagger}$ & $2.05 \pm 1.31 \quad(0.78-6.66)$ & $1.29 \pm 0.59 \quad(0.64-2.49)^{\dagger}$ \\
\hline Hemoglobin $(\mathrm{g} / \mathrm{d} l)$ & $14.9 \pm 1.6 \quad(13.0-19.0)$ & $11.2 \pm 2.0 \quad(7.4-16.0)^{\$}$ & $12.0 \pm 1.5 \quad(9.26-14.0)^{\$}$ \\
\hline Total bilirubin $(\mathrm{mg} / \mathrm{d} l)$ & $1.13 \pm 0.39(0.5-2.0)^{*}$ & $2.96 \pm 2.31 \quad(0.7-8.7)^{*}$ & $17.57 \pm 8.60 \quad(10-40)$ \\
\hline Direct bilirubin $(\mathrm{mg} / \mathrm{d} l)$ & $0.22 \pm 0.08(0.1-0.4)^{*}$ & $1.59 \pm 1.48 \quad(0.2-4.6)^{*}$ & $12.52 \pm 5.60 \quad(6.4-26)$ \\
\hline AST (IU/l) & $28.7 \pm 9.1 \quad(19-54)$ & $76.6 \pm 48.6(27-264)^{\$}$ & $101.0 \pm 63.1(35-231)^{\$}$ \\
\hline $\mathrm{ALT}(\mathrm{IU} / l)$ & $32.5 \pm 15.4(14-60)$ & $83.6 \pm 119.3(26-587)$ & $140.1 \pm 114.6(36-432)^{\$}$ \\
\hline GGT $(\mathrm{IU} / l)$ & $37.1 \pm 23.6(12-84)^{*}$ & $107.7 \pm 182.8(13-879)^{*}$ & $687.3 \pm 584.5(33-1,850)$ \\
\hline Child-Pugh score & - & $9.0 \pm 2.9 \quad(6-14)$ & - \\
\hline $\begin{array}{l}\text { Proportion of persons with } \\
\text { hepatic encephalopathy }(\%)\end{array}$ & - & 20.7 & - \\
\hline
\end{tabular}

Statistical significance test was done by Scheffe's test. *: $p<0.05$ vs. obstructive jaundice, ${ }^{\dagger}: p<0.05$ vs. liver cirrhosis, $\$$ : $p<0.05$ vs. control, \&: $p<0.05$ by chi-square test. Data are mean \pm standard deviation (range).

MnB: blood manganese level. AST: aspartate aminotransferase. ALT: alanine aminotransferase. GGT: $\gamma$-glutamyltransferase.

cirrhosis from the hepatology clinic of the same hospital. A diagnosis of hepatitis B-induced liver cirrhosis was established using patient questionnaires combined with clinical, laboratory, endoscopic and ultrasonographic findings ${ }^{10)}$. The 16 healthy controls were selected from healthy persons visiting our hospital for comprehensive health check ups; these subjects had no evidence of anemia or liver disease. None of the patients in the three groups had a history of exposure to Mn. All subjects provided their written informed consent, and the study protocol was approved by the Institutional Review Board of our hospital.

Questionnaires, laboratory testing, and analysis of Mn concentration

Samples of heparinized venous blood were collected from each patient. Each patient was administered a questionnaire that recorded basic demographic information as well as information about smoking history, alcohol consumption, medications, and recent medical history. Each subject was asked about his or her previous disease history in detail. Laboratory tests, designed to detect liver dysfunction or anemia, consisted of liver function tests as well as hematological investigations, such as blood cell count, hemoglobin, and hematocrit. The severity of liver disease was assessed using Child/ Pugh scores ${ }^{13)}$, which included the variables of ascites, encephalopathy, serum albumin, serum bilirubin, and prothrombin time, with each variable scored from 1 to 3 points for increasing abnormality.

Blood Mn concentrations were determined by flameless graphite furnace atomic absorption spectrophotometry (AAS; Spectra AA880-GTA 100, Varian, Australia) ${ }^{14,15}$. Aliquots $(0.1 \mathrm{ml})$ of blood were diluted 20 fold with $0.1 \%$ $\mathrm{v} / \mathrm{v}$ Triton X-100, and 15- $\mu l$ samples were injected into the graphite furnace.

\section{$M R I$}

MRI examinations were performed using a 1.5 Tesla system (Signa Horizon LX; GE Medical Systems; Milwaukee, WI, USA). The spin echo (SE) technique was applied with two acquisitions in the T1-weighted sequence and one acquisition in the T2-weighted sequence. T1-weighted images were acquired in the axial and sagittal planes using the following parameters: 466/ 14 (repetition time $\left(\mathrm{T}_{\mathrm{R}}\right)$ /echo time $\left(\mathrm{T}_{\mathrm{E}}\right) \mathrm{ms}$ ] two excitations, a $22-\mathrm{cm}$ field-of-view, a $256 \times 256$ matrix, and a slice thickness of $7 \mathrm{~mm}$. T1-weighted sagittal 


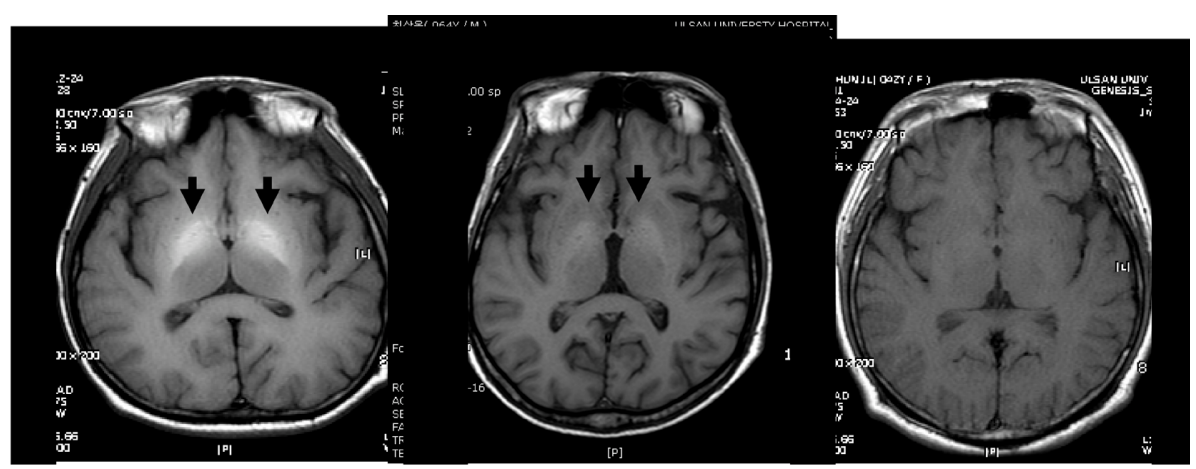

A

B

C

Fig. 1. Axial T1-weighted MR image of a patient with liver cirrhosis (A), a patient with biliary obstruction (B), and a healthy control (C). Black arrows show markedly increased signal intensities (A), and slightly increased signal intensities (B) at the globus pallidus.

images were also obtained using $\mathrm{T}_{\mathrm{R}} / \mathrm{T}_{\mathrm{E}}=466 / 14 \mathrm{~ms}$ with the remaining parameters as listed above. Axial T2weighted $\left(T_{R} / T_{E}=4,000 / 100 \mathrm{~ms}\right)$ images were also obtained. The signal intensity of the GP relative to that recorded from frontal white matter was subjectively evaluated by a radiologist without prior knowledge of patient status. For quantitative evaluation of signal intensities, the region of interest (ROI) was placed in the regions of highest intensities in the GP and subcortical frontal white matter by visual assessment, and the PI was calculated as the ratio of signal intensity in the GP to that in the subcortical frontal white matter multiplied by $100^{8)}$.

\section{Statistical analyses}

Mean values of continuous variables for the three groups were compared using one way analysis of variance (ANOVA). If the ANOVA showed significance at $p<0.05$, Scheffe's multiple comparison test was performed to identify the subgroup that was significantly different from the other subgroups. The differences in frequency distribution of males, drinkers, and persons with increased signals were ascertained by the chi-square test.

\section{Results}

The general, clinical and biochemical characteristics of the subjects are shown in Table 1. The mean ages of biliary obstructive patients, cirrhotic patients, and normal controls were $66.8 \mathrm{yr}, 47.5 \mathrm{yr}$ and $53.8 \mathrm{yr}$, respectively. Patients with biliary obstruction had mean total and direct bilirubin concentrations of $17.57 \mu \mathrm{g} / \mathrm{d} l$ and $12.52 \mu \mathrm{g} / \mathrm{d} l$, respectively, each of which differed significantly from those of the cirrhotic and healthy control groups. $\gamma$ Glutamyltransferase activity also showed a similar pattern. Levels of hemoglobin, aspartate aminotransferase, and alanine aminotransferase in patients with liver cirrhosis and biliary obstruction were significantly different from those in healthy controls. Patients with biliary obstruction had a mean blood Mn of $1.29 \mu \mathrm{g} / \mathrm{d} l$, similar to that of healthy controls, but was significantly different from that of cirrhotic controls. The mean PI of patients with biliary obstruction was similar to that of healthy controls, but differed significantly from that of cirrhotic controls. A slight increase in pallidal signal was observed in one biliary obstructive patient, with a total bilirubin concentration of $40 \mu \mathrm{g} / \mathrm{d} l$, but in none of the healthy controls (Fig. 1). However, increased pallidal signals in the GP were observed in 25 of 29 $(86.2 \%)$ patients with liver cirrhosis $(p<0.05)$. In patients with liver cirrhosis, there was a significant correlation between blood Mn and PI $(\gamma=0.575, p<0.01)$ and between Child/Pugh scores and PI $(\gamma=0.419, p<0.05)$, but not between blood $\mathrm{Mn}$ and bilirubin concentrations. The correlations between PI and blood Mn and between PI and bilirubin were not statistically significant in the biliary obstructive patients.

\section{Discussion}

Increases in blood Mn levels and pallidal signals in cirrhotic patients are likely to be multifactorial. A significant relationship has been observed between pallidal T1 signal intensity and severity of liver lesions, as assessed by Child/Pugh scores ${ }^{10,16-18)}$. However, Child/ Pugh scores are a complex of heterogeneous variables, some of which are related indirectly to portal-systemic shunt (e.g. ascites and encephalopathy) and others to biliary excretion (e.g. bilirubin). Significant relationships have also been observed between pallidal T1 signal intensity and the presence of portal hypertension/portalsystemic shunt ${ }^{16,17,19,20)}$, and between pallidal T1 signal intensity and the severity of liver lesions as well as portal- 
systemic shunt ${ }^{10,16,17)}$.

To our knowledge, the present work is the first study of patients with bile duct obstruction, but without portalsystemic shunts. Mean blood Mn level and pallidal T1 signals were not increased in these patients, but were increased in patients with liver cirrhosis. Although patients with bile duct obstruction had concentrations of total and direct bilirubin much higher than those in patients with liver cirrhosis, almost all had incomplete biliary obstructions, suggesting that Mn might be excreted via the bile. One patient with biliary obstruction and a high pallidal signal had a total bilirubin concentration of $40 \mu \mathrm{g} / \mathrm{d} l$, indicative of nearly total biliary obstruction. Our results suggest that incomplete biliary obstruction alone may not increase blood $\mathrm{Mn}$ and pallidal signals. Pallidal signals are significantly decreased after liver transplantation, even in patients with chronic graft rejection and severe cholestasis but no evidence of cirrhosis ${ }^{17)}$. Moreover, there was no correlation between pallidal T1 signals and the presence of cholestatic liver disease ${ }^{17)}$. Our findings, coupled with these earlier results ${ }^{17}$, suggest that the crucial mechanisms responsible for high pallidal signals are not linked to the impairment of bile excretion in liver cirrhosis.

High pallidal T1 signals have been reported in children with congenital abnormalities of portal-systemic shunt due to dysgenesis of the portal venous system, but who showed almost normal liver function ${ }^{21,22)}$. High pallidal signals have also been observed in patients with long standing portal-systemic shunt alone, due to portal vein thrombosis, without liver cirrhosis ${ }^{23)}$. Animal models of portal-systemic shunt without cirrhosis have higher concentrations of $\mathrm{Mn}$ in the basal ganglia than in animal models of cirrhosis ${ }^{24)}$. Moreover, high pallidal T1 signals were more prominent in patients with idiopathic portal hypertension than in patients with liver cirrhosis ${ }^{25}$, showing that there is no correlation between pallidal T1 hyperintensity and the severity of liver dysfunction or hepatic encephalopathy. Taken together, these findings suggest that portal-systemic shunt per se may be a major determinant in the etiology of pallidal T1 signals. Since the liver plays an important role in regulating the distribution and excretion of Mn, portal-systemic shunt probably decreases the hepatobiliary clearance of $\mathrm{Mn}$, resulting in increased blood Mn levels along with high pallidal T1 signals ${ }^{26)}$.

Our study had its limitation. Almost all patients had partial biliary obstruction of subchronic duration, although patients with acute obstructions, such as acute cholangitis, were excluded. Chronic and complete biliary obstruction may affect the intensity of the pallidal signal.

In summary, our findings indicate that portal-systemic shunt, rather than obstruction of biliary excretion, may be responsible for the increased blood Mn concentration and pallidal T1 signal observed in patients with chronic liver diseases.

Acknowledgments: This work was funded by Ulsan University Hospital (Biomedical Research Center Promotion Fund; UUH-2006-04).

\section{References}

1) Newland MC, Ceckler TL, Kordower JH and Weiss B: Visualizing manganese in the primate basal ganglia with magnetic resonance imaging. Exp Neurol 106, 251-258 (1989)

2) Nelson K, Golnick J, Korn T and Angle C: Manganese encephalopathy: utility of early magnetic resonance imaging. Br J Ind Med 50, 510-513 (1993)

3) Mirowitz SA, Westrich TJ and Hirsch JD: Hyperintense basal ganglia on T1-weighted MR images in patients receiving parenteral nutrition. Radiology 181, 117-120 (1991)

4) Ejima A, Imamura T, Nakamura S, Saito H, Matsumoto $\mathrm{K}$ and Momono S: Manganese intoxication during total parental nutrition. Lancet 339, 426 (1992)

5) Butterworth RF, Spahr L, Fontaine $S$ and Layrargues GP: Manganese toxicity, dopaminergic dysfunction and hepatic encephalopathy. Metab Brain Dis 10, 259-267 (1995)

6) Hauser RA, Zesiewicz TA, Rosemurgy AS, Martinez $\mathrm{C}$ and Olanow CW: Manganese intoxication and chronic liver failure. Ann Neurol 36, 871-875 (1994)

7) Saito $\mathrm{H}$ and Ejima A: Liver dysfunction and probable manganese accumulation in the brainstem and basal ganglia. J Neurol Neurosurg Psychiatry 58, 760-761 (1995)

8) Krieger D, Krieger S, Jansen O, Gass P, Theilmann L and Lichtnecker H: Manganese and chronic hepatic encephalopathy. Lancet 346, 270-274 (1995)

9) Maeda H, Sato M, Yoshikawa A, Kimura M, Sonomura T, Terada $\mathrm{M}$ and Kishi K: Brain MR imaging in patients with hepatic cirrhosis: relationship between high intensity signal in basal ganglia on T1-weighted images and elemental concentrations in brain. Neuroradiology 39, 546-550 (1997)

10) Park NH, Park JK, Choi Y, Yoo C-I, Lee CR, Lee H, Kim HK, Kim S-R, Jung TH, Park J, Yoon CS and Kim Y: Whole blood manganese correlates with high signal intensities on T1-weighted MRI in patients with liver cirrhosis. Neurotoxicology 24, 909-915 (2003)

11) Morgan MY: Cerebral magnetic resonance imaging in patients with chronic liver disease. Metab Brain Dis 13, 273-290 (1998)

12) Choi Y, Park JK, Park NH, Shin JW, Yoo C-I, Lee CR, Lee H, Kim HK, Kim S-R, Jung T-H, Park J, Yoon CS and Kim Y: Whole blood and red blood cell manganese reflected signal intensities of T1-weighted MRI better than plasma manganese in liver cirrhotics. J Occup Health 47, 68-73 (2005)

13) Pugh RNH, Murray-Lyon IM, Dawson JL, Pietroni, MC and Williams R: Transection of the esophagus for bleeding esophageal varices. Br J Surg 60, 646-649 (1973) 
14) Baldwin S, Deaker M and Maher W: Low volume microwave digestion of marine biological tissues for the measurement of trace elements. Analyst 119, 17011704 (1994)

15) Francois B, Oliver G, Josiane A and Francis P: Determination of manganese in biological materials by electrothermal atomic absorption spectrometry, a review. Clinical Chem 34, 227-234 (1988)

16) Kulisevsky J, Pujol J, Balanzo J, Junque C, Deus J, Capdevilla A and Villanueva C: Pallidal hyperintensity on magnetic resonance imaging in cirrhotic patients: clinical correlations. Hepatology 16, 1382-1388 (1992)

17) Pujol A, Pujol J, Graus F, Rimola A, Peri J, Mercader JM, Garcia-Pagan JC, Bosch J, Rodes J and Tolosa E: Hyperintense globus pallidus on T1-weighted MRI in cirrhotic patients is associated with severity of liver failure. Neurology 43, 65-69 (1993)

18) Taylor-Robinson SD, Oatridge A, Hajnal JV, Burroughs AK, McIntyre N and deSouza NM: MR imaging of the basal ganglia in chronic liver disease: correlation of T1-weighted and magnetisation transfer contrast measurements with liver dysfunction and neuropsychiatric status. Metab Brain Dis 10, 175-188 (1995)

19) Weissenborn K, Ehrenheim C, Hori A, Kubicka S and Manns MP: Pallidal lesions in patients with liver cirrhosis: clinical and MRI evaluation. Metab Brain Dis 10, 219-232 (1995)

20) Thuluvath PJ, Edwin D, Yue NC, deVilliers C, Hochman $S$ and Klein $A$ : Increased signals seen in globus pallidus in T1-weighted magnetic resonance imaging in cirrhotics are not suggestive of chronic hepatic encephalopathy. Hepatology 21, 440-442 (1995)
21) Yanai S, Minami T, Sonoda K, Gondo K, Tasaki K, Hijii T, Fukushige J, Ueda K, Hirata T, Hayashi T and Hasuo K: Patent ductus venosus associated with a hyperintense globus pallidum on T1-weighted magnetic resonance imaging and pulmonary hypertension. Eur J Pediatr 154, 526-529 (1995)

22) Ihara K, Hijii T, Kuromaru R, Ariyoshi M, Kira R, Fukushige $\mathrm{J}$ and Hara T: High-intensity basal ganglia lesions on T1-weighted images in two toddlers with elevated blood manganese with portosystemic shunts. Neuroradiology 41, 195-198 (1999)

23) Nolte W, Wiltfang J, Schindler CG, Unterberg K, Finkenstaedt M, Niedmann PD, Hartmann $\mathrm{H}$ and Ramadori G: Bright basal ganglia in T1-weighted magnetic resonance images are frequent in patients with portal vein thrombosis without liver cirrhosis and not suggestive of hepatic encephalopathy. J Hepatol 29, 443-449 (1998)

24) Rose C, Butterworth RF, Zayed J, Normandin L, Todd K, Michalak A, Spahr L, Huet PM and PomierLayrargues G: Manganese deposition in basal ganglia structures results from both portal-systemic shunting and liver dysfunction. Gastroenterology 117, 640-644 (1999)

25) Fukuzawa T, Matsutani S, Maruyama H, Akiike T, Saisho $\mathrm{H}$ and Hattori T: Magnetic resonance images of the globus pallidus in patients with idiopathic portal hypertension: a quantitative analysis of the relationship between signal intensity and the grade of portosystemic shunt. J Gastroenterol Hepatol 21, 902-907 (2006)

26) Papavasiliou PS, Miller ST and Cotzias CC: Role of liver in regulating distribution and excretion of manganese. Am J Physiol 211, 211-216 (1966) 